\title{
An Experimental Study on the Incentives of the Probabilistic Serial Mechanism
}

\author{
David Hugh-Jones ${ }^{1} \quad$ Morimitsu Kurino ${ }^{2} \quad$ Christoph Vanberg $^{3}$
}

\begin{abstract}
We report an experiment on the Probabilistic Serial (PS) mechanism for allocating indivisible goods. The PS mechanism, a recently discovered alternative to the widely used Random Serial Dictatorship mechanism, has attractive fairness and efficiency properties if people report their preferences truthfully. However, the mechanism is not strategy-proof, so participants may not truthfully report their preferences. We investigate misreporting in a set of simple applications of the PS mechanism. We confront subjects with situations in which the theory suggests that there is an incentive or no incentive to misreport. We find little misreporting in situations where misreporting is a Nash equilibrium. However, we also find a significant degree of misreporting in situations where there is actually no benefit to doing so. These findings suggest that the PS mechanism may have problems in terms of truthful elicitation.
\end{abstract}

Keywords: Probabilistic serial mechanism; incentives

JEL classification: C78, C91, C92, D47 


\section{Introduction}

In the last decade, market designers have explored real-life applications of indivisible goods resourse allocation problems when monetary transfers are not allowed. Examples include oncampus housing for college students [2], the assignment of seats in public schools to pupils [3], kidney transplantation for patients [20], and course seats to college students [22,6].

In such problems, it is often wasteful to require fairness ex post, since items cannot be divided. ${ }^{4}$ Thus, randomization is commonly used to ensure fairness ex ante. The simplest problem is the socalled house allocation problem $[16,5]$ where $n$ indivisible goods, called houses, are to be allocated to $n$ agents without monetary transfers, and each agent consumes exactly one house. An (ordinal) mechanism is a systematic way of assigning houses to agents for each reported ordinal preference profile. $^{5}$ The most popular mechanism in theory and practice is the random serial dictatorship mechanism (RSD), which randomly orders agents and then lets them successively choose their favorite houses among those available. Bogomolnaia and Moulin [5] point out that RSD may be inefficient from an ex ante perspective. Specifically, they show that RSD induces a distribution over final allocations that is not ordinally efficient. This means that there is another way to randomly allocate the houses which would be unanimously preferred by all participants.

Bogomolnaia and Moulin propose the probabilistic serial mechanism (PS) as an alternative to RSD because PS achieves ordinal efficiency. In addition, PS satisfies the fairness property of envy-freeness [12], which has played a central role in resource allocation problems. ${ }^{6}$ RSD is not envy-free. These considerations suggest that RSD, which is widely used in real-world markets, could be better replaced by PS.

However, the attractive properties of PS assume that participants truthfully reveal their preferences, i.e., their rankings over the houses to be allocated. If participants misreport their rankings, these properties may not hold. And indeed, a problematic feature of PS is that it may induce incentives for participants to misreport. In technical terms, these concerns are about the strategyproofness of an allocation mechanism. A strategy-proof mechanism does not allow any agent to benefit from misrepresenting her preference regardless of others' reporting. PS is not fully strategy-proof because in some situations some agents may benefit from misreporting their preferences. Even where such incentives are absent, the mechanism is less transparent than RSD, and thus it is possible that human participants may falsely perceive incentives to misreport. ${ }^{7}$

\footnotetext{
${ }^{4}$ For example, see Kesten and Yazici [18].

${ }^{5}$ In this paper we focus on ordinal mechanisms rather than cardinal mechanisms that elicit cardinal utilities. There are three common justifications for the ordinal approach as explained in Hashimoto, Hirata, Kesten, Kurino, and Ünver [15]: First, since agents are boundedly rational, cardinal preferences are difficult to elicit. Second, ordinal mechanisms are simpler and more practical than cardinal ones. Third, real-life matching markets function mostly through the elicitation of ordinal preferences.

${ }^{6} \mathrm{~A}$ random assignment is envy-free if no agent prefers anybody else's assignment regardless of her cardinal utilities.

${ }^{7}$ Note that the mechanism we consider is ordinal. A mechanism is strongly strategy-proof if for each agent the
} 
In this paper, we report a laboratory experiment investigating how often agents misrepresent their preferences under PS. We implement a situation where subjects have an incentive to misrepresent their preferences. We also implement situations where subjects do not benefit from misrepresenting their preferences, but may mistakenly believe that they do. We find little strategic misrepresentation in the former situation, but high levels of misrepresentation in the latter. The phenomenon is robust to experience and feedback.

\section{The Probabilistic Serial Mechanism}

We first introduce the model and then describe the random serial dictatorship mechanism (RSD) and the probabilistic serial mechanism (PS). The random assignment problem, also called a house allocation problem, is the allocation problem where $n$ indivisible objects (henceforth "houses") are to be allocated to $n$ agents, each agent is to receive exactly one house, and no monetary transfers are permitted $[16,5]$. Let $I=\{1, \ldots, n\}$ be the set of agents and $A$ the set of houses. Each agent $i \in I$ has a (cardinal) utility $u_{i, a}$ from being assigned each house $a$ and is not indifferent among two distinct houses. Let us denote utilities of agent $i$ by $u_{i}:=\left(u_{i, a}\right)_{a \in A}$ and a utility profile by $u:=\left(u_{i}\right)_{i \in I}$. Agent $i$ with utilities $u_{i}$ has a strict preference $\succ_{i}$ over houses. We say that $u_{i}$ is consistent with a strict preference $\succ_{i}$ on $A$ when for all houses $a, b \in A, a \succ_{i} b \Leftrightarrow$ $u_{i, a}>u_{i, b}$. We fix $I$ and $A$ throughout the paper.

We consider the situation where probabilistic assignments are possible. A random allotment for some agent $\boldsymbol{i}$ is a probability distribution on houses, $P_{i}:=\left(p_{i, a}\right)_{a \in A}$, where $p_{i, a}$ denotes the probability that agent $i$ receives house $a$. Thus, for each $a \in A, p_{i, a} \in[0,1]$ and $\sum_{a \in A} p_{i, a}=1$. A random assignment is a collection of random allotments for all agents, denoted by $P=\left(P_{i}\right)_{i \in A}$, such that for each house $a \in A, \sum_{i \in I} p_{i, a}=1$. In other words, a random assignment is a bistochastic matrix. A deterministic assignment specifies which house each agent is assigned such that no two distinct houses are assigned to an agent. That is, it is a random assignment with entries of either 0 or 1.8

Each agent $i$ evaluates a given random assignment $P=\left(P_{i}\right)_{i \in I}$ as the expected utility of her random allotment, $\sum_{a \in A} p_{i, a} u_{i, a}$.

We focus on ordinal mechanisms as they are used in real-life markets. An ordinal mechanism

induced random assignment under truthful reporting (first-order) stochastically dominates the one under misreporting: equivalently, for all cardinal utilities consistent with truthful preferences, the expected payoff under truthful reporting is greater than or equal to the one under misreporting. PS is weakly strategy-proof in the following sense: for each agent the induced random assignment under truthful reporting is not stochastically dominated by the one under misreporting. However, as in our experiment, agents with certain cardinal utilities may gain from misreporting their preferences. A recent paper by Kojima and Manea [19] shows that in a large but finite economy where there is a sufficiently large supply of each kind of object, PS becomes strategy-proof.

${ }^{8}$ Note that to implement a random assignment we need to have a probability distribution over deterministic assignments - a lottery. A lottery induces the random assignment by taking the weighted sum of deterministic assignments. The converse is also true: any random assignment can be implemented by a lottery [4, 23]. Thus we focus on random assignments. 
is a systematic way of selecting a random assignment for each preference profile. Here we assume that there is a central authority which asks each agent $i \in I$ to report her ordinal preference $\succ_{i}$, and uses a mechanism $\varphi$ to select a random assignment $\varphi(\succ)$ based on the reported preference profile $\succ$.

The random serial dictatorship mechanism (RSD) selects the random assignment, denoted by $R S D(\succ)$, for a preference profile as follows. First, an ordering of agents is randomly drawn with equal probability. An ordering is denoted by $\left(i_{1}, i_{2}, \ldots, i_{n}\right)$ where $i_{1}$ is the first agent, $i_{2}$ the second agent, and so on. Second, for each realized ordering $\left(i_{1}, \ldots, i_{n}\right)$, the first agent $i_{1}$ is assigned her favorite (most preferred) house according to $\succ_{i_{1}}$, the second agent $i_{2}$ is assigned her favorite house among the remaining ones according to $\succ_{i_{2}}$, and so on.

Example 1. We illustrate how to compute the RSD outcome. This example is used in our experiment. There are three agents $(1,2,3)$ and three houses $(a, b$ and $c)$ with preferences as given in the following table.

$$
\begin{array}{l|lll}
\succ_{1} & a & c & b \\
\succ_{2} & a & b & c \\
\succ_{3} & b & a & c
\end{array}
$$

For example, the ordering $(2,3,1)$ is drawn with a probability of $1 / 6$. Under this ordering, the first agent 2 gets her favorite house $a$, the second agent 3 gets her favorite house $b$ among the remaining houses $b, c$, and the last agent 1 gets the remaining house $c$. The outcome is shown below.

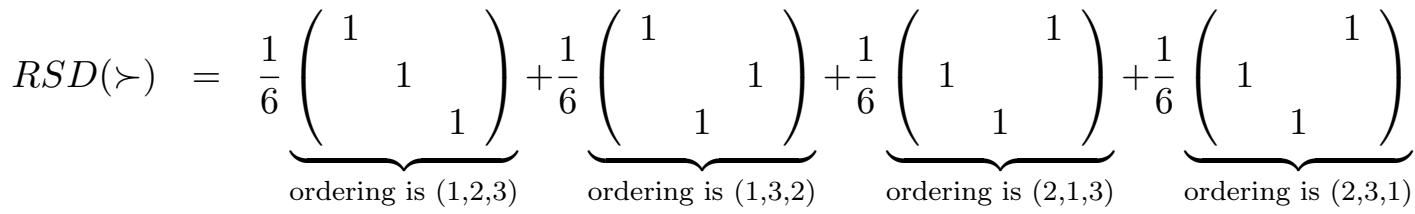

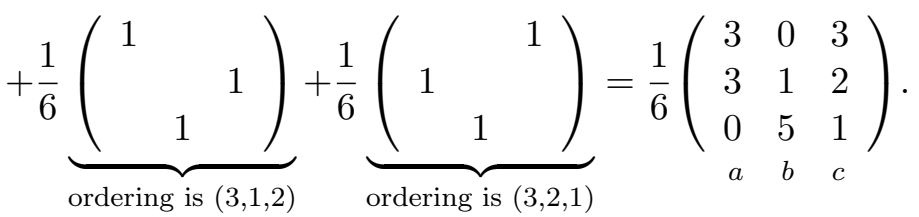

$\diamond$

The probabilistic serial mechanism (PS) [5] selects the random assignment by the following simultaneous eating algorithm: Given a preference profile $\succ$, think of each house as possessing an infinitely divisible unit of probability, which will be given to all agents from time 0 to 1 .

Step 1: Each agent receives the probability of her favorite house at the same unit speed. Proceed to the next step when a house is completely exhausted.

Step $s$ : Each agent receives the probability of her remaining favorite house at the same unit speed. Proceed to the next step when a house is completely exhausted. 
The algorithm stops when each agent has been given exactly 1 total unit of probability (i.e., at time 1). The random allotment of an agent $i$ by $P S$ is then given by the amount that she was given from each house before the algorithm stopped. Let $P S(\succ)$ be the random assignment of PS for problem $\succ$.

Example 2. We illustrate how to compute the PS outcome. Consider the same preference profile as in Example 1. In Step 1, agents 1 and 2 receive the probability of their favorite house $a$, while agent 3 gets the probability of her favorite house $b$. At time $1 / 2, a$ is exhausted and $1 / 2$ of $b$ is available. Then, in Step 2, all agents turn to their next favorite among the remaining houses $b, c$. That is, agent 1 receives the probability of her house $c$, while agents 2 and 3 receive the probability of house $b$. At time $t=1 / 2+1 / 4=3 / 4, b$ is exhausted and the amount of $3 / 4$ of $c$ is available. In the last step, all agents receive the probability of the remaining house $c$. Thus,

$$
P S(\succ)=\underbrace{\left(\begin{array}{cc}
\frac{1}{2} & \\
\frac{1}{2} & \\
& \frac{1}{2}
\end{array}\right)}_{\text {amounts received in Step } 1}+\underbrace{\left(\begin{array}{cc} 
& \frac{1}{4} \\
\frac{1}{4} & \\
\frac{1}{4} &
\end{array}\right)}_{\text {amounts received in Step } 2}+\underbrace{\left(\begin{array}{rr}
\frac{1}{4} \\
\frac{1}{4} \\
\frac{1}{4}
\end{array}\right)}_{\text {amounts received in Step } 3}=\left(\begin{array}{ccc}
\frac{1}{2} & 0 & \frac{1}{2} \\
\frac{1}{2} & \frac{1}{4} & \frac{1}{4} \\
0 & \frac{3}{4} & \frac{1}{4}
\end{array}\right) .
$$

\subsection{Efficiency}

We turn to efficiency notions that rely only on ordinal preferences. A deterministic assignment $D$ is Pareto efficient at $\succ$ if there is no deterministic assignment $D^{\prime}$ such that for each $i \in I$, $D^{\prime} \succeq_{i} D$ and for some $i \in I, D^{\prime} \succ_{i} D$. A random assignment $P$ is $\boldsymbol{e x}$ post efficient at $\succ$ if it assigns positive probability only to Pareto efficient deterministic assignments.

Ex post efficiency is a weak condition, since a large set of deterministic assignments are Pareto efficient. Bogomolnaia and Moulin [5] introduce an appealing ex ante notion of ordinal efficiency based on first-order stochastic dominance: Consider two random allotments $P_{i}$ and $Q_{i}$ of agent $i$. Then, $P_{i}$ (first-order) stochastically dominates $Q_{i}$ at $\succ_{i}$, denoted by $P_{i} \succeq_{i}^{s d} Q_{i}$ if for all $a \in A, \sum_{b \in A: b \succeq_{i} a} p_{i, b} \geq \sum_{b \in A: b \succ_{i} a} q_{i, b}$. In other words, for any set of $i$ 's "most preferred" alternatives, $P_{i}$ assigns at least as great a total probability to that set as $Q_{i}$ does. If the inequality is strict for at least one $a \in A$, we say that $P_{i}$ strictly stochastically dominates $Q_{i}$ at $\succ_{i}$, denoted by $\succ_{i}^{s d}$. Note that $\succeq_{i}^{s d}$ is a partial order. Moreover, $\succeq_{i}^{s d}$ has a characterization in terms of cardinal utilities: $P_{i} \succeq_{i}^{s d} Q_{i}$ if and only if $u_{i}\left(P_{i}\right) \geq u_{i}\left(Q_{i}\right)$ for any utility $u_{i}$ consistent with $\succ_{i}$. Thus, stochastic dominance is a sufficient (but not necessary) condition for agent $i$ to prefer the allotment $P_{i}$ to $Q_{i}$.

Now we are ready to formalize ordinal efficiency. A random assignment $P$ stochastically dominates $Q$ at $\succ$ if for each $i \in I, P_{i} \succeq_{i}^{s d} Q_{i}$ and for some agent $i \in I, P_{i} \succ_{i}^{s d} Q_{i}$. A random assignment $P$ is ordinally efficient at $\succ_{i}$ if it is not dominated by another random assignment. 
Finally, a mechanism is ordinally efficient (ex post efficient) if it selects an ordinally efficient (ex post efficient) random assignment for each preference profile. Ordinal efficiency is the strongest concept in our ordinal environment.

Although it is ex post efficient, RSD is not ordinally efficient, as the following example shows.

Example 3. ${ }^{9} I=\{1,2,3,4\}$ and $A=\{a, b, \ldots\}$.

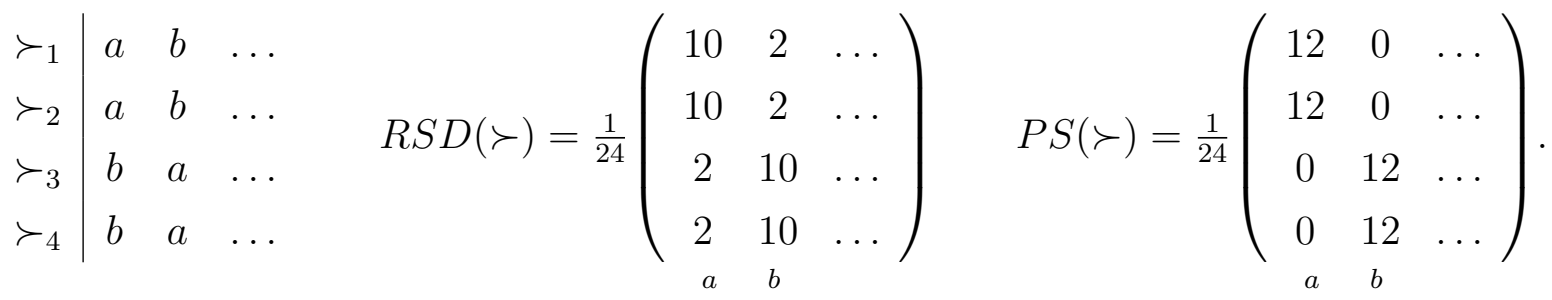

Here, under RSD, agent 1 gets house $b$ if agent 2 is chosen first and she is chosen second, with a probability of 1 in 12 . Under PS, agents 1 and 2 each receive half of house $a$ while agents 3 and 4 each receive half of house $b$. The random assignment $R S D(\succ)$ is not ordinally efficient, as it is stochastically dominated by $P S(\succ)$ at $\succ$. Note that for each $\succ^{\prime} \in \mathbf{P}^{I}$, while $P S\left(\succ^{\prime}\right)$ is ordinally efficient, in general $P S\left(\succ^{\prime}\right)$ does not always stochastically dominate $R S D\left(\succ^{\prime}\right)$ at $\succ^{\prime}$ [5].

To understand the substance of these properties, it may be helpful to consider their implications in this example. The fact that the random assignment $P S(\succ)$ stochastically dominates the random assignment $R S D(\succ)$ means that all four agents in this example would prefer $P S(\succ)$ to $R S D(\succ)$ ex ante, regardless of their cardinal utilities. Although no mutually advantageous trades would be possible after either assignment is "realized," all agents would therefore agree ex ante to a shift from RSD to PS as a mechanism in this case. ${ }^{10}$

\subsection{Incentive compatibility}

A mechanism $\varphi$ induces a preference revelation game where each agent is equipped with the set of preferences as her strategy set. Thus, a reported preference as a strategy may or may not coincide with the agent's true one. Hence, if somebody is insincere, PS's desirable properties of ordinal efficiency and envy-freeness may be achieved not at the truthful preference profile but at the reported profile. For this reason, it is important for a mechanism to elicit truthful preferences from the agents.

For expositional purposes, we reserve the notation $\succ$ to denote a truthful preference profile, and $\succ_{i}^{\prime}$ to denote an arbitrary preference of agent $i$. Similarly we use $u$ to denote a truthful utility

\footnotetext{
${ }^{9}$ This example is from Bogomolnaia and Moulin [5].

${ }^{10}$ Kesten [17] shows that we can always achieve a PS assignment after allowing agents to engage in specific trades (based on Gale's top trading cycles [21]) starting from the equal division of probabilistic shares.
} 
profile, and $u_{i}^{\prime}$ an arbitrary utility function of agent $i$. Now we are ready to introduce our notion of incentive compatibility. We denote the set of all strict preferences by $\mathbf{P}$.

Under a mechanism $\varphi$, a preference profile $\succ^{\prime}$ is a Nash equilibrium under $u$ if for each $i \in I$ and each $\succ_{i}^{\prime \prime} \in \mathbf{P}, u_{i}\left[\varphi\left(\succ^{\prime}\right)\right] \geq u_{i}\left[\varphi\left(\succ_{i}^{\prime \prime}, \succ_{-i}^{\prime}\right)\right]$.

A mechanism $\varphi$ is strategy-proof if for each $i \in I$, each $\succ \in \mathbf{P}^{I}$, and each $\succ_{i}^{\prime} \in \mathbf{P}, \varphi_{i}(\succ) \succeq_{i}^{s d}$ $\varphi_{i}\left(\succ_{i}^{\prime}, \succ_{-i}\right)$. It is straightforward to see that under a non-strategy-proof mechanism, it is not a Nash equilibrium under some utility profile for each agent to report a truthful preference. ${ }^{11} \mathrm{We}$ will examine this case for PS in the next section.

\subsection{Related literature}

To our knowledge, we are the first to report a laboratory experiment on the PS mechanism. There are several experimental studies on a slightly different problem, called a house allocation problem with existing tenants [2], where some of the agents, existing tenants, have property rights to houses. The widely used mechanism in this problem is random serial dictatorship with squatting rights, where existing tenants may either keep their current houses or enter the RSD mechanism. Chen and Sönmez [7] and Chen and Sönmez [8] report experimental studies on RSD with squatting rights, as well as some new mechanisms, in incomplete information and complete information environments respectively. As our experimental environment involves complete information, Chen and Sönmez [8] is closely related to our paper. In their design, which has three agents and two houses with an outside option, $100 \%$ of the subjects revealed their preferences truthfully. Guillen and Kesten [14] report an experiment on other mechanisms in the problem with existing tenants.

\section{Experimental Design}

We implement the PS and RSD mechanisms in a laboratory experiment. Our aim is to assess the extent to which individuals truthfully report their preferences under PS. For this purpose, we design the simplest possible environment which captures the incentive issues of PS. As will become clear, the RSD treatment was implemented mainly as a control in order to explore the motivations underlying the behavior observed under PS. For this reason our analysis will focus on results under the PS mechanism.

Subjects play in groups of three, and three houses $(a, b, c)$ are allocated within each group. Utility profiles are induced through monetary payments. Subjects participate in four games involving different payoff profiles, given in the four tables below, under complete information. Two of these games are symmetric (Sym-H and Sym-L), and two are asymmetric (Asym-H and Asym-L). In ordinal terms, the two symmetric and the two asymmetric games are identical. As we will see, however, incentives differ between these games because the value of the second best option differs.

\footnotetext{
${ }^{11}$ By definition, $\varphi$ is strategy-proof if and only if for each preference profile $\succ$ and each utility profile $u$ consistent with $\succ, \succ$ is a Nash equilibrium under $u$. See Ekici and Kesten [10] for more equilibrium analysis on PS.
} 
Table 1: Games played by subjects

\begin{tabular}{|c|c|ccc|}
\multicolumn{7}{c}{ Sym-H } \\
\hline Player & Situation & $a$ & $b$ & $c$ \\
\hline 1 & SH1 & 100 & 95 & 0 \\
2 & SH1 & 100 & 95 & 0 \\
3 & SH1 & 100 & 95 & 0 \\
\hline
\end{tabular}

\begin{tabular}{|c|c|ccc|}
\multicolumn{5}{|c}{ Sym-L } \\
\hline Player & Situation & $a$ & $b$ & $c$ \\
\hline 1 & SL1 & 100 & 30 & 0 \\
2 & SL1 & 100 & 30 & 0 \\
3 & SL1 & 100 & 30 & 0 \\
\hline
\end{tabular}

Asym-H

\begin{tabular}{|c|c|ccc|}
\hline Player & Situation & $a$ & $b$ & $c$ \\
\hline 1 & AH1 & 100 & 0 & 95 \\
2 & AH2 & 100 & 95 & 0 \\
3 & AH3 & 95 & 100 & 0 \\
\hline
\end{tabular}

Asym-L

\begin{tabular}{|c|c|ccc|}
\hline Player & Situation & $a$ & $b$ & $c$ \\
\hline 1 & AL1 & 100 & 0 & 30 \\
2 & AL2 & 100 & 30 & 0 \\
3 & AL3 & 30 & 100 & 0 \\
\hline
\end{tabular}

${ }^{\dagger}$ Note: 1 point $=15$ UK pence. In Sym-H, Sym-L and Asym-L, sincerity is Nash. In Asym-H the unique Nash equilibrium has AH3 falsely declaring $a \succ b \succ c$.

Agents can find themselves in one of eight situations in total (two in the symmetric games, six in the asymmetric games). We give these situations the labels SH1 et cetera, as in Table 1, and refer to the agent in the relevant situation as "agent SH1" et cetera.

The rationale behind the games' construction is as follows. Under PS, the Sym-H and Sym-L games create no incentive to misreport one's preferences. The Asym-H game, by contrast, gives one agent (AH3) an incentive to misreport his or her preferences, assuming the other agents report truthfully. As explained below, Asym-H may also create a false perception by another agent (AH1) that s/he should misreport his/her preferences. The Asym-L game is a control, allowing us to check that the behavior in Asym- $\mathrm{H}$ is indeed related to the incentives created by the valuable second best option.

\subsection{Benchmark predictions}

We derive benchmark predictions for PS and RSD. Since RSD is strategy-proof, the following result is apparent, and is stated without proof.

Proposition 1. Under the random serial dictatorship mechanism (RSD), truthful reporting constitutes a weakly dominant strategy for all agents, and truthful reporting by all agents is a Nash equilibrium in all four games.

Next we examine the Nash equilibria of PS. Let us denote the utility profiles under the tables Sym-H, Sym-L, Asym-H, and Asym-L by $u^{S H}, u^{S L}, u^{A H}$, and $u^{A L}$. The utility profiles $u^{S H}$ and $u^{S L}$ are consistent with the ordinal preference profile $\succ^{S}$, where for each agent $i, a \succ_{i}^{S} b \succ_{i}^{S} c$. Under this preference profile, truthful reporting induces the same random assignment under both PS and RSD: 


$$
P S\left(\succ^{S}\right)=\left(\begin{array}{ccc}
a & b & c \\
1 / 3 & 1 / 3 & 1 / 3 \\
1 / 3 & 1 / 3 & 1 / 3 \\
1 / 3 & 1 / 3 & 1 / 3
\end{array}\right)
$$

For both of these symmetric utility profiles, truthful reporting by all agents constitutes the unique Nash equilibrium under PS. ${ }^{12}$

Next, consider the asymmetric utility profiles $u^{A H}$ and $u^{A L}$, both of which are consistent with the ordinal preference profile in Example 1, denoted by $\succ^{A S}$. Under this preference profile, truthful reporting under PS induces the following random assignment whose calculation is given in Example 3.

$$
P S\left(\succ^{A S}\right)=\left(\begin{array}{ccc}
\frac{1}{2} & 0 & \frac{1}{2} \\
\frac{1}{2} & \frac{1}{4} & \frac{1}{4} \\
0 & \frac{3}{4} & \frac{1}{4}
\end{array}\right) .
$$

Suppose instead that agent 3 reports a false preference to swap her top two houses as $a \succ_{3}^{\prime} b \succ_{3}^{\prime}$ $c$, while agents 1 and 2 continue to report truthfully. This would induce the random assignment

$$
P S\left(\succ_{3}^{\prime}, \succ_{-3}^{A S}\right)=\left(\begin{array}{ccc}
\frac{1}{3} & 0 & \frac{2}{3} \\
\frac{1}{3} & \frac{1}{2} & \frac{1}{6} \\
\frac{1}{3} & \frac{1}{2} & \frac{1}{6}
\end{array}\right) .
$$

By misreporting her preference, agent 3 decreases the probability of obtaining either her worst $(c)$ or best $(b)$ house, in favor of her middle house $(a)$. In expected utility terms, this deviation is attractive if her middle house is sufficiently close in value to her most preferred, as is the case under utility profile $u^{A H}$. Indeed, the following calculation demonstrates that misreporting is a best response for agent $\mathrm{AH} 3$ if the others report truthfully:

$u_{3}^{A H}\left[P S_{3}\left(\succ^{A S}\right)\right]=0 \cdot 95+\frac{3}{4} \cdot 100+\frac{1}{4} \cdot 0=75<\frac{1}{3} \cdot 95+\frac{1}{2} \cdot 100+\frac{1}{6} \cdot 0=81.66=u_{3}^{A H}\left[P S_{3}\left(\succ_{3}^{\prime}, \succ_{-3}^{A S}\right)\right]$.

As a consequence, truthful reporting by all three agents is not a Nash equilibrium under utility profile $u^{A H}$. Closer analysis reveals that the outcome where only agent AH3 misreports by switching her top two choices constitutes the unique Nash Equilibrium under profile $u^{A H}$. Under $u^{A L}$, truthful reporting by all three agents is a Nash equilibrium, since in this case agent AL3 does not value her middle house as highly. ${ }^{13}$ We summarize these theoretical results in the following proposition.

\footnotetext{
${ }^{12}$ This result is verified by computation.

${ }^{13}$ There exists a further equilibrium in which agent AL3 deviates by swapping her worst two options. However, this strategy yields the same outcome.
} 
Proposition 2. Consider the probabilistic serial mechanism (PS).

1. In the two symmetric games (Sym-H and Sym-L), truthful reporting by all three agents constitutes the unique Nash equilibrium.

2. In the asymmetric game in which the middle houses are highly valued (Asym-H)

(a) truthful reporting by all agents is not a Nash equilibrium, since agent AH3 can profitably deviate by switching her top two choices,

(b) the preference profile where only agent AH3 misreports by switching her top houses constitutes the unique Nash equilibrium.

3. In the asymmetric game in which the middle houses have less value (Asym-L), there are two Nash equilibria whose PS outcomes are the same: One is truthful reporting by all agents, and another is only agent AL3 misreports by switching her worst two houses.

Proof. We have already verified Part 2(b), whose proof is from Bogomolnaia and Moulin [5]. The other parts are verified by computation.

Based on Propositions 1 and 2, we formulate the following benchmark predictions.

HYPOTHESIS 1: Under RSD, subjects will report truthfully in all situations.

HYPOTHESIS 2: Under PS,

1. Subjects will report their true preferences in all situations except situation AH3.

2. In situation AH3, subjects will misreport their preferences by swapping the ranking of their top two houses.

\subsection{Alternative predictions}

We have seen that theory suggests subjects should benefit from misreporting only in situation AH3 (if others report truthfully) under PS. Despite this, it is conceivable that subjects might falsely perceive an incentive to deviate. As an example, consider an agent in situation AH1. This agent gets his top payoff from house $a$, but also gets a high payoff from house $c$. House $a$ is ranked first or second by both of the other agents. If agent AH1 expects the other two agents to report sincerely, it may therefore appear sensible for agents in situation AH1 to (falsely) rank house $c$ first, avoiding competition for house $a$. The intuition underlying this perception is that house $c$ is a "bird in the hand," and that one risks losing it if one goes for option $a$ "in the bush." In fact, this intuition is mistaken: in the unique Nash equilibrium, where agent AH2 is sincere and agent AH3 is insincere, agent AH1 ought to be sincere. Agent AH1 should also be sincere if both other agents are sincere. These facts can be verified by computation. The correct intuition is that one increases one's total probability of winning either $a$ or $c$ by going for house $a$ first.

A similar intuition applies to agents in situation SH1. Here, any agent might consider ranking house $b$ first in order to avoid competition for $a$. However, it is easy to verify that this would not be a good idea given sincere reporting by the other agents. In fact, sincerity by agent 1 is always optimal unless one of the other agents declared $(b, c, a)$, ranking her best house last. 
Although these intuitions are false in the context of PS, we suspect that they are quite compelling, as they do apply in other situations where people compete to obtain scarce houses. In many such situations, there is indeed an advantage to avoiding competition for the most popular option in order to obtain a highly valued alternative. In the school choice context, when the non-strategy-proof Boston mechanism was used for the Boston Public Schools system before 2004, experts often advised parents to place less popular schools high on their list in order to improve their chances of getting into these schools [3,1]. This strategy is beneficial in so many contexts that one might conjecture the existence of a 'don't chase the popular girl' heuristic. ${ }^{14}$ If such a heuristic exists, individuals may employ it in PS even when this is not beneficial. If so, this would be bad news for PS. In contrast, it is quite apparent that the intuition does not apply in the context of RSD, as there is no 'competition' when agents sequentially choose. Indeed, it should be relatively easy to see that sincerity is always optimal in RSD: when a participant's turn comes to choose, it never makes sense to state false preferences. These considerations lead us to formulate the following hypothesis.

HYPOTHESIS 3a: Under PS, subjects in situations SH1 and AH1 will misreport by switching their top two houses. This behavior is most likely to occur in situation AH1, since there the middle house is both highly valued and not desired by either of the remaining agents.

Note that the behavior predicted in Hypothesis 3a is inconsistent with the theoretical equilibrium predictions. If this behavior occurs within the experiment, the best responses for other agents in the relevant games may be different from those predicted in equilibrium. It is therefore interesting to ask what the best responses to these deviations from equilibrium play would be.

First, consider situation SH1. As explained above, sincerity is a best response in this situation as long as neither of the other agents declare $(b, c, a)$, ranking the best house $(a)$ worst. In this sense, sincerity in situation SH1 is robust to deviations from equilibrium play. Next, consider game Asym-H. If agent AH1 reports $(c, a, b)$, as Hypothesis 3a predicts, then truth-telling is always a best response for agents AH2 and AH3. Thus, the benchmark prediction concerning insincerity in situation AH3 is sensitive to the deviation from equilibrium play predicted in Hypothesis 3a. This will be important to keep in mind if we should find substantial amounts of misreporting in situation AH1.

HYPOTHESIS 3b: Under PS, if substantial misreporting occurs in situation AH1, we expect truthful reporting in situation AH3.

\footnotetext{
${ }^{14}$ This might explain the notoriously non-Nash scene of the film A Beautiful Mind, in which John Nash encourages his friends to divide their attention among different girls.
} 


\subsection{Experimental Procedures}

All experiments were conducted at a computer laboratory at the University of Warwick. Subjects were students from a variety of disciplines. We ran four sessions implementing PS, and two sessions implementing RSD. ${ }^{15}$ Within a session, subjects were randomly assigned to matching groups of size six. Each session consisted of 48 rounds. At the start of each round, subjects were randomly matched to form groups of three, always with others from within their respective matching groups. ${ }^{16}$ This procedure allows us to treat each matching group of six as a statistically independent observation. In each of the 48 rounds, subjects were presented with a payoff table as presented above, and asked to submit a ranking over the houses. All sessions involved the same sequence of payoff tables, with each of the eight situations repeated six times. At the end of a round, the reported rankings were used to determine the random assignment of the mechanism in effect. Subjects then received feedback concerning this random assignment only (not the realized deterministic assignment). After all 48 rounds were completed, one round was chosen for payment. For this round, the random assignment was played out in order to determine a realized deterministic assignment. Subjects were paid privately. They received 15 pence for each point value of the house assigned to them, plus a $£ 5$ showup fee. The mean payment, including showup fee, was $£ 15.46$. Sessions lasted 60-90 minutes. Experiments were programmed in z-Tree [11].

Since PS is unfamiliar and unintuitive, an important feature of our design is the way in which we explained the algorithm to subjects. In designing the instructions we aimed to make sure that subjects understood the mechanism, while avoiding any suggestion as to whether or not it was individually advantageous to report truthfully. To do so, we demonstrated PS using a graphical animation. In this animation, the three available objects were represented as houses, within each of which a "pie" of probability was displayed. The animation demonstrated the algorithm being executed step by step, with the pies being sequentially filled in until all pies were fully allocated. The instructions allowed subjects to click through each step of the animation at their own pace. Each step was accompanied by an explanatory text. ${ }^{17}$ Subsequently, the same animation was shown (more quickly) at the end of each round to display the consequences of the subjects' actual choices. We did not include a comprehension test, since we expected that an intuitive understanding of the mechanism would not correlate well with answering technical questions about it, and we thought that the vast majority of subjects would be unable to calculate outcomes from inputs by hand, without an impracticable amount of training. Arguably, our experiment had clearer instructions, more repetitions and more feedback than typical real-world assignment mechanisms such as school lotteries. Despite this, it is plausible that subjects would have difficulty fully understanding the mechanism, in the sense of failing to correctly predict the consequences of reporting truthfully or

\footnotetext{
${ }^{15}$ The PS sessions were conducted in January 2010. The RSD sessions were conducted in May 2012.

${ }^{16}$ Subjects were not aware of the exact design of matching groups; they were simply told that they would be rematched "at random."

${ }^{17} \mathrm{~A}$ screencast is available at https://sites.google.com/site/davidhughjones/instructions\%20screencast.ogv
} 

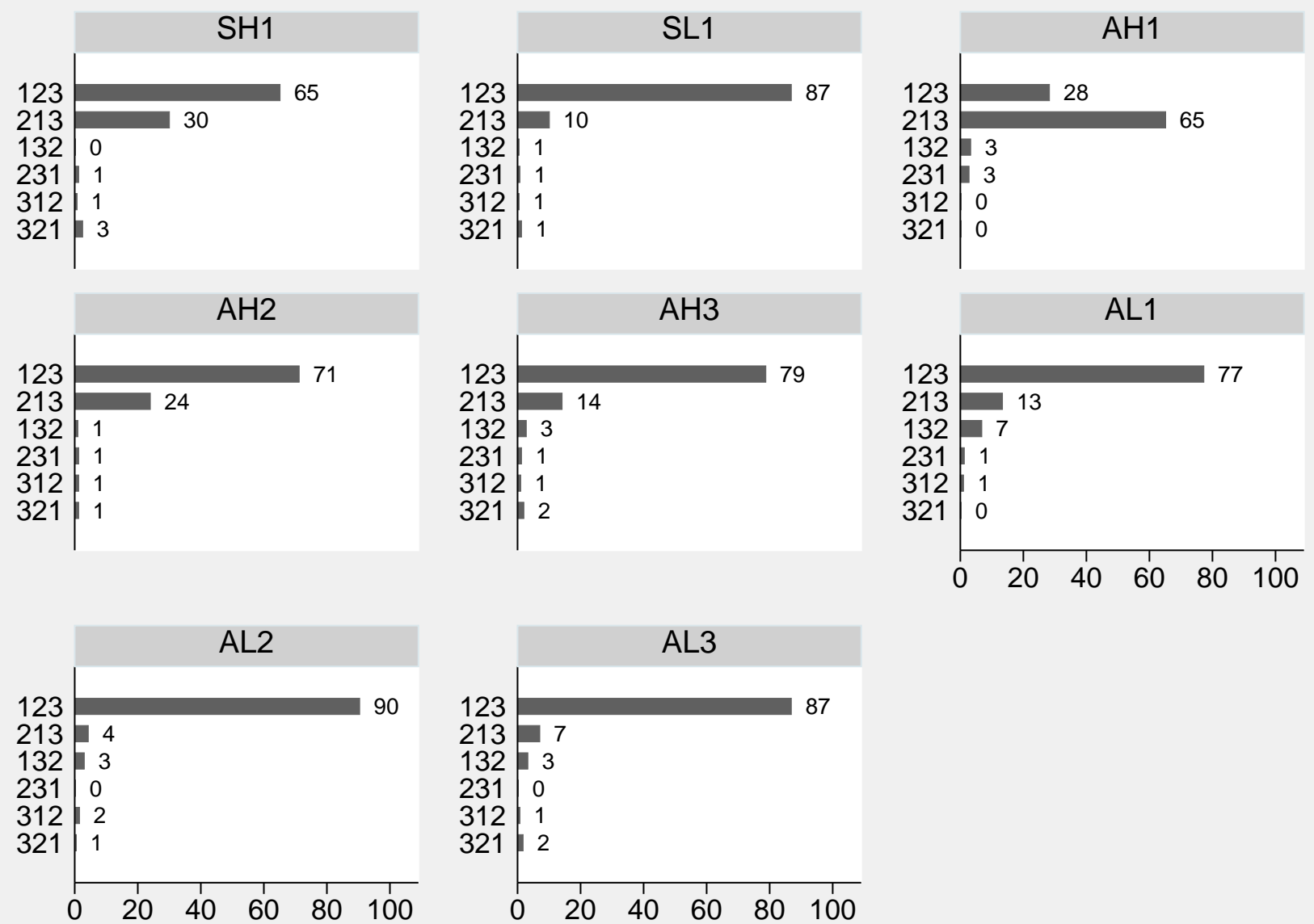

Figure 1: Frequency of strategies by situation under PS

Note: Each three-digit code represents the placement of the options truly ranked 1st, 2nd, and 3rd. For example, strategy 123 is "sincere" while strategy 213 means the top two options were switched.

not. Indeed, hypothesis 3a is based on our expecting such confusion, and one of the experiment's purposes is to test precisely this. ${ }^{18}$

\section{Results}

\subsection{Sincerity under the PS mechanism}

Our data comprises choices of rankings over three houses made by subjects in eight distinct situations: SH1, SL1, AH1, AH2, AH3, AL1, AL2, AL3 (see Table 1). Within each situation, our main interest is in comparing the ranking that subjects report to their true ranking in that situation. To facilitate comparisons across situations, we adopt the following convention in labeling the six possible rankings that may be reported. For each situation, the ranking " 123 " will be the sincere ranking for that situation, the ranking "213" will denote the ranking that results from

\footnotetext{
${ }^{18}$ Guillen and Hing [13] argue that confusion may be an important factor in matching experiments, and show that subjects' decisions can be strongly affected by both correct and incorrect advice.
} 
Table 2: Percentage of non-sincere reports formed by switching top two houses (PS)

\begin{tabular}{rr|rrrrrrrr}
\hline & & \multicolumn{8}{c}{ situation } \\
& Total & SH1 & SH2 & AH1 & AH2 & AH3 & AL1 & AL2 & AL3 \\
\hline top switches & $78 \%$ & $86 \%$ & $77 \%$ & $91 \%$ & $83 \%$ & $67 \%$ & $59 \%$ & $45 \%$ & $54 \%$ \\
N non-sincere & 852 & 138 & 52 & 284 & 114 & 84 & 90 & 38 & 52
\end{tabular}

exchanging the top two options relative to the sincere ranking, etc. For example, consider situation AL1. For subjects in this situation we have $a \succ c \succ b$. Iff a subject reported $b \succ c \succ a$, this would be coded as "321". Using this notation, Figure 1 shows the distribution of the reported rankings in each situation, for subjects participating in the PS sessions. Although these figures involve repeated observations from the same subjects and matching groups, they suggest a number of clear patterns in behavior.

The first discernible pattern is that situation AH1 involves by far the least sincere reporting ("123"), with only $28 \%$. A second pattern is that non-sincere behavior appears to be more common in the "H" situations, i.e., those where the payoff difference between the top two choices is small. For example, subjects appear to be more sincere in situation SL1 (87\%) than in situation SH1 (65\%). Third, another pattern observable at this aggregate level is that the most common nonsincere ranking is " 213 ". That is, subjects who report non-sincere rankings most often reverse the ranking of their top two choices. Quite sensibly, subjects rarely try to game the mechanism by falsely ranking their least preferred option. ${ }^{19}$ Table 2 shows the percentage of non-sincere reports formed by switching the top two options. Especially in those situations where non-sincere behavior is the most common (SH1, AH1, AH2, AL1), the vast majority of false rankings are formed by swapping the two top choices.

These data appear to support Hypothesis 3a: insincere behavior occurs most often in situation AH1, followed by situations SH1 and AH2. In contrast, Hypothesis 2 appears doubtful, as we observe a non-negligible frequency of misreporting in all situations, and situation AH3 does not seem to elicit significantly more non-sincere behavior. If Hypothesis $3 \mathrm{a}$ is accepted, then the latter pattern would be consistent with Hypothesis 3b. That is, perhaps the unexpectedly large percentage of sincere behavior in situation AH3 was supported by the unexpected non-sincerity in situation AH1. In order to assess the statistical significance of the various comparisons underlying these conclusions, we conduct non-parametric tests using the matching groups as independent units of observation. Figure 2 shows the overall frequencies of sincere reporting in each situation, separately for each of the 11 matching groups participating in our PS sessions. For any given pair of situations, say SH1 and SL1, we have 11 independently measured pairs of frequencies. These data can be used to compare sincerity between situations using a Wilcoxon signed-rank test. ${ }^{20}$

\footnotetext{
${ }^{19}$ The fact that we observe this behavior at all may be attributable to experimentation or confusion.

${ }^{20}$ This is a nonparametric paired-sample test. In our case, the members of each matching group are observed in two conditions. The test is based on counting the number of groups for whom the fraction of sincere reports is larger
} 

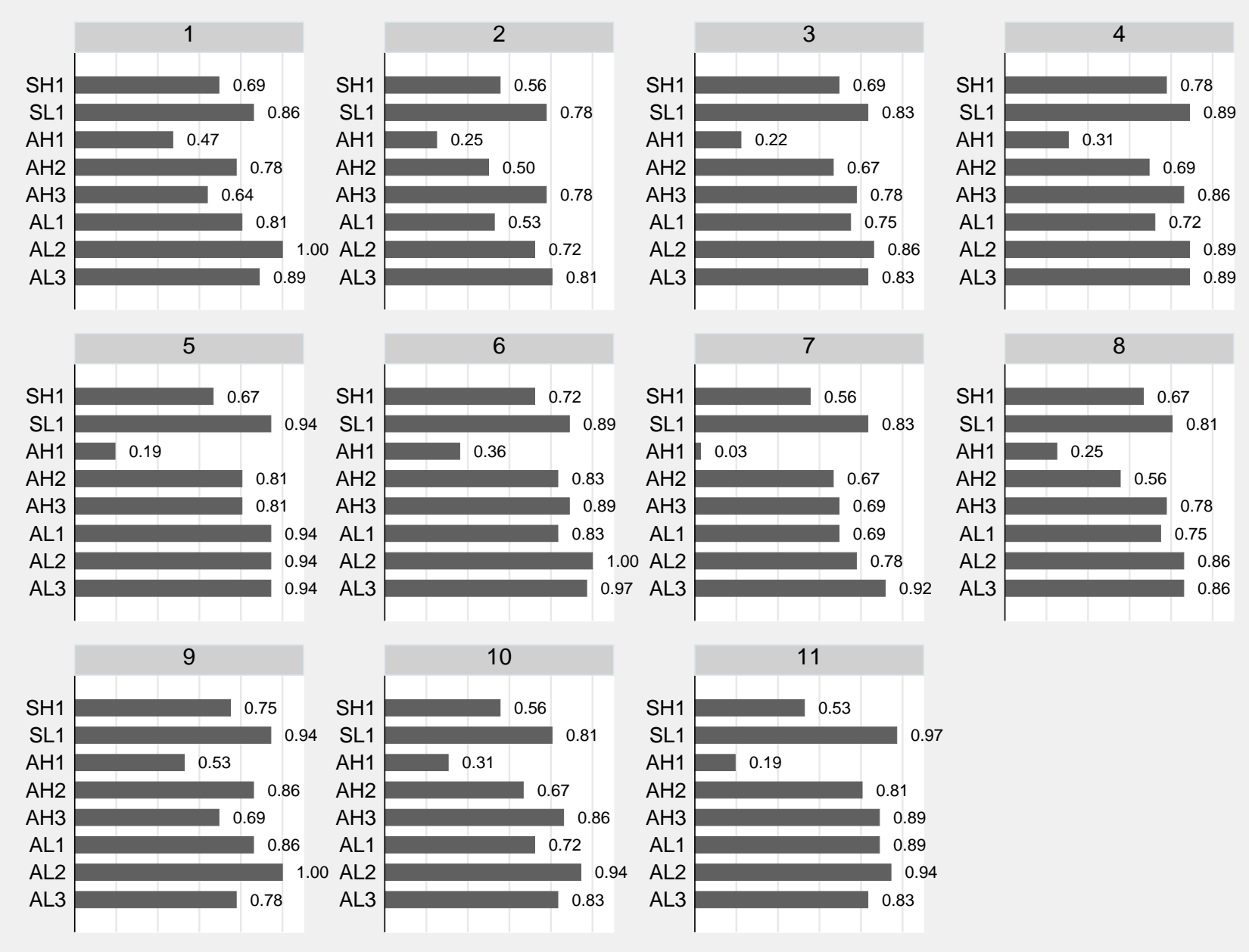

Figure 2: PS: Frequencies of sincere reporting by situation and matching group

Note: Each bar in these graphs represents 36 observations from a single matching group of six individuals. (Each individual is observed six times in each situation.)

Returning to Hypothesis 2, this is indeed clearly rejected. In eight of 11 matching groups, situation AH3 elicits significantly more sincere behavior than any other " $H$ " situation (i.e., a situation in which the middle option is worth 95 points). (Wilcoxon signed-rank test: $p<0.01$ on comparisons to SH1 and AH1, and $p<0.1$ on comparison with AH2.) Thus we conclude:

Result 1. Hypothesis 2 is rejected: Under PS, non-sincere reporting occurs in all situations. Moreover, situation AH3 (i.e., the one in which theory predicts misreporting) elicits more sincere behavior than situations SH1, AH1 and AH2 (for which theory predicts sincerity).

Next, we turn to Hypothesis 3a, which predicts misreporting in situations SH1 and AH1. We have already noted that the aggregate pattern seems to strongly support the second part of this hypothesis. And indeed, signed-rank tests show that situation AH1 elicits less sincere reporting

in one condition than in the other. The central assumption is that the measurements are statistically independent between the groups. 
than any other situation, with $p<0.01$ in all seven comparisons. In fact, situation AH1 involves the least sincere reporting across all situations within each and every matching group. For situation SH1, we find significantly more misreporting than in all other situations except AH1 $(p<0.01$ on all comparisons except to situation $\mathrm{AH} 2$, for which $p=0.08)$. Thus we can confidently conclude the following.

Result 2. Hypothesis $3 a$ is supported: Under PS, non-sincere behavior is significantly more common in situation AH1 than in any other situation, followed by situation SH1.

Finally, consider the prediction that misreporting will be more common when the top options are similar in value, i.e., in the "H" situations, than when they are far apart, that is, in the "L" situations. The four relevant comparisons are situations (SL1 vs. SH1), (AL1 vs. AH1), (AL2 vs. AH2), and (AL3 vs. AH3). All of these differences go in the hypothesized direction and are highly significant. (Wilcoxon signed-rank test: $p<0.01$ for the first three, and $p=0.02$ for the last comparison.) This leads us to conclude:

Result 3. Under PS non-sincere behavior is significantly more common in situations where the top two choices are similar in value.

Together these results lend support to Hypothesis 3a. It appears that, contrary to theoretical predictions, subjects in our experiment systematically misreported their preferences in a situation in which they had no incentive to do so. In contrast, our rejection of Hypothesis 2 suggests that subjects may have reported their preferences sincerely in a situation where theory suggests they should do otherwise. However, the latter result must be interpreted in light of our Hypothesis 3b, which is consistent with the data. That is, given that in a majority of cases agent AH1 reported non-sincerely, we can rationalize agent AH3's high levels of sincerity as a best response.

Given the strong support we have found for Hypothesis 3a, we next turn to exploring possible explanations for why this type of insincere reporting occurs. One possibility is that insincerity in situation AH1 is a best response to observed behavior. This explanation, however, can be quite easily dismissed. It can be verified that it is optimal for agent AH1 to swap her top two preferences only if one of the other agents declared $(c, b, a)$, putting their third preference first. But agent AH2 or AH3 declared $(c, b, a)$ in only $1 \%$ of observed cases (9 out of 792 ).

Since AH1's insincerity is not a best response, it is natural to ask whether it disappears over time. Figure 3 investigates this question by graphing the frequency of sincere behavior in each situation, over the six repetitions of the situation in which each subject is placed. While in other situations, insincerity appears to decrease, it does not do so in situation AH1. Individuallevel regressions confirm this first impression: insincerity is not significantly predicted either by repetition from 1 to 6 , or by time period from 1 to $48 .^{21}$

\footnotetext{
${ }^{21}$ Details are contained in the appendix.
} 


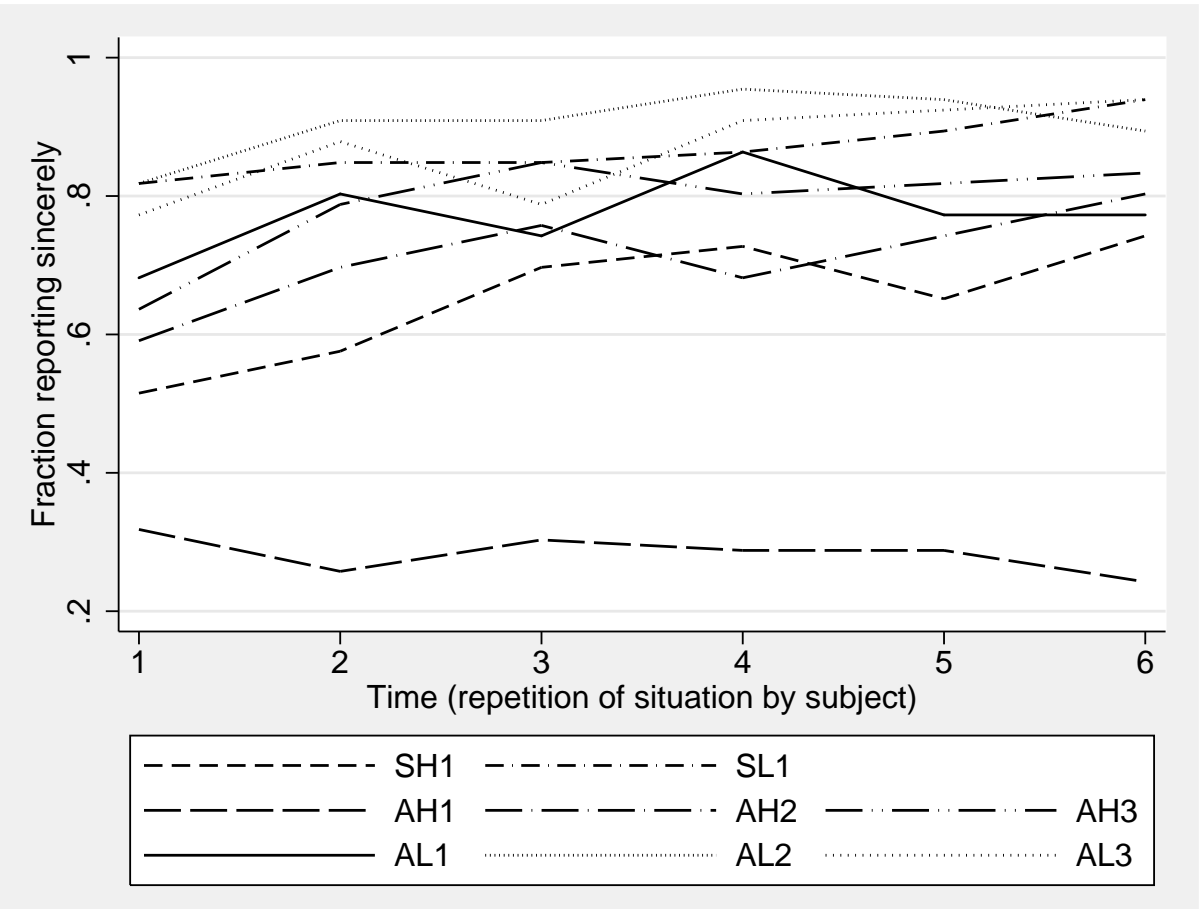

Figure 3: Frequencies of sincere reporting by situation over time under PS

Note: The time index refers to the number of repetitions of each situation. Each subject is in each situation six times. The frequency reported is the fraction of subjects reporting truthfully the n-th time they are in that situation. (Thus it combines decisions that are not actually made simultaneously, as when Tina is in situation AH1 for the third time in period 20, and Tom in period 25.)

A potentially important alternative hypothesis is that subjects are intentionally "settling for" their second best option in situation AH1, in order to allow the other subjects to obtain their first choices for sure. If subjects in roles AH2 and AH3 report sincerely, then the subject in role AH1 can choose to switch her top two choices, in which case she gets her second choice (worth 95) for sure and the other subjects each get their first choices (worth 100) for sure. If instead she reports sincerely, the resulting lottery gives her an equal chance of obtaining her top two choices and each of the other subjects has a $25 \%$ chance of obtaining their worst option. Thus, observed behavior might be explained by social preferences, rather than by the behavioral heuristic that we posit.

This alternative hypothesis can be evaluated by comparing behavior under PS to that observed under RSD. Specifically, if insincere reporting in situation AH1 is driven by social preferences, we should expect the same to occur under RSD. For again, by switching her top choices, a subject can secure her second choice and ensure that other subjects each get their first choices. By reporting sincerely, she would again get an equal chance of her top two choices, while agent AH2 would get her worst option with a probability of $50 \%$ and agent AH3 would get her worst option with probability one in six. In the next section, we report results from the two sessions which implemented RSD, and test this explanation. 

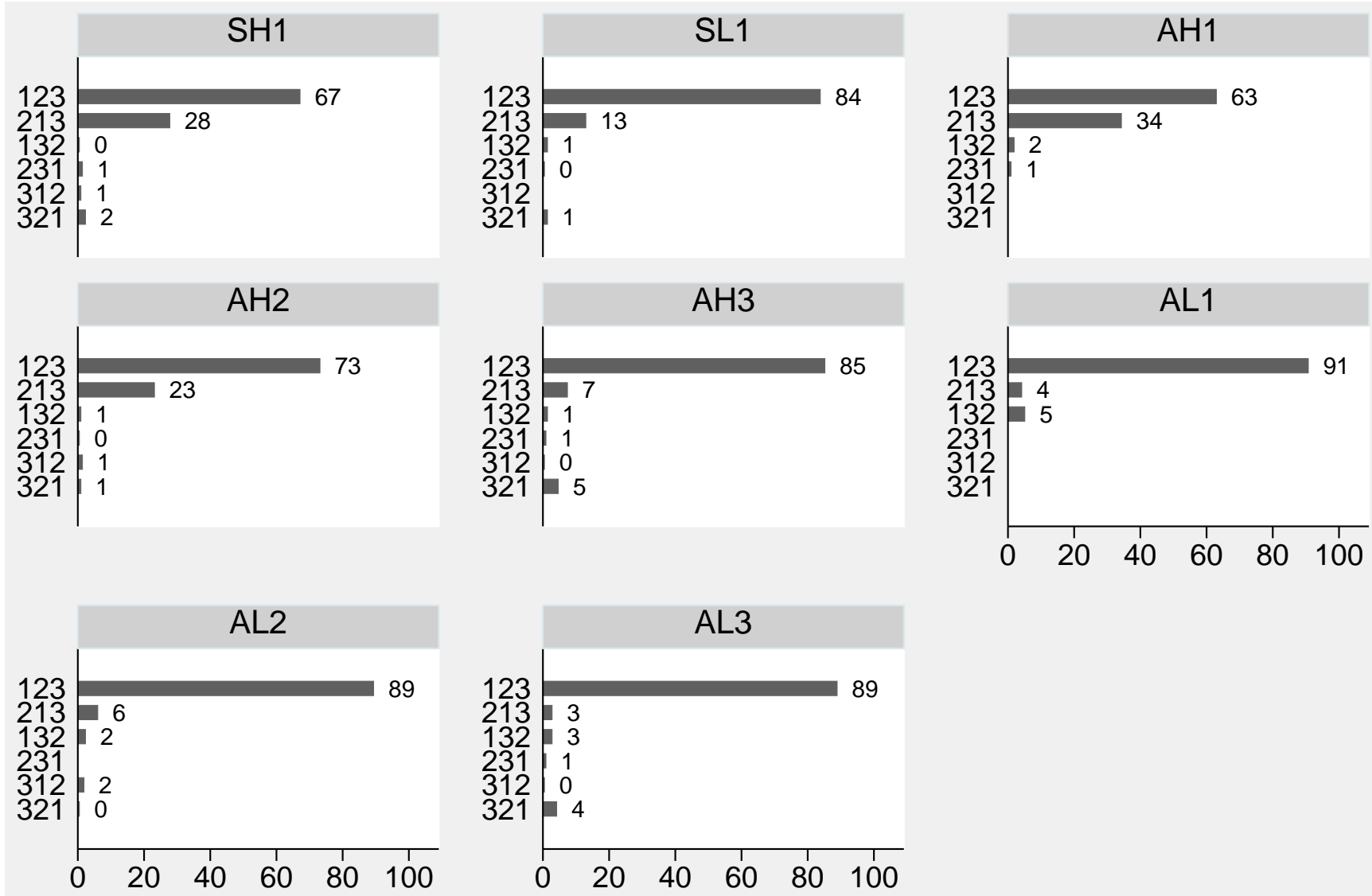

Figure 4: Frequency of strategies by situation under RSD

Note: Each three-digit code represents the placement of the options truly ranked 1st, 2nd, and 3rd. For example, strategy 123 is "sincere" while strategy 213 means the top two options were switched.

\subsection{Sincerity under the RSD mechanism}

As indicated earlier, incentives to misreport are transparently absent under RSD. Therefore we expect little insincere reporting under this mechanism (Hypothesis 1). Using the notation previously introduced, Figure 4 shows the distribution of the reported rankings observed in the two RSD sessions we conducted. Perhaps surprisingly, we do see a non-negligible amount of misreporting even under this simple mechanism.

Result 4. Hypothesis 1 is rejected: A non-negligible percentage of subjects report their preferences non-sincerely under RSD.

As under PS, non-sincere behavior is more common in situation AH1 than in all other situations. These differences are significant for comparisons of AH1 to SL1, AH3, AL1, AL2 and AL3 (Wilcoxon signed-rank tests: $p<0.05$ in each case), but not compared to situations SH1 or AH2 $(p=0.3$ in both cases $) .{ }^{22}$

\footnotetext{
${ }^{22}$ As in the previous subsection, tests are based on session-level percentages of sincere behavior in each situation. See Figure 5 for the raw data underlying these tests.
} 


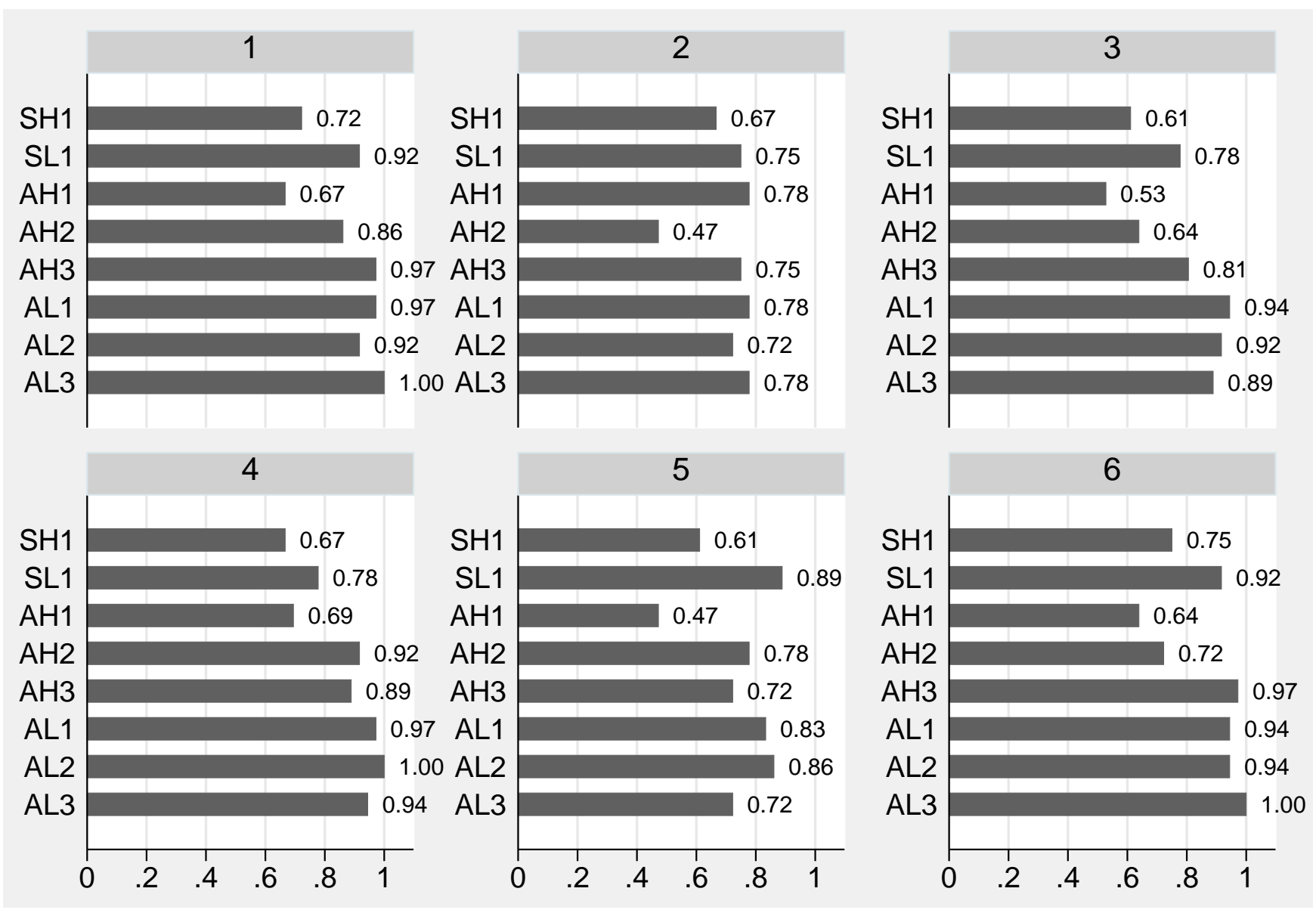

Figure 5: RSD: Frequencies of sincere reporting by situation and matching group

Note: Each bar in these graphs represents 36 observations from a single matching group of six individuals. (Each individual is observed six times in each situation.)

Result 5. Under RSD, non-sincere behavior is significantly more common in situation AH1 than in most other situations, followed by situation SH1.

Given the transparent absence of strategic incentives to misreport under RSD, this result suggests that some subjects in situation AH1 deliberately settle for their second choice in order to allow others to obtain their first. However, the frequency of such misreporting is much smaller under RSD (37\%) than under PS (72\%). This difference is highly significant (Wilcoxon rank-sum test, $p<0.01$ ), suggesting that social preferences play at most a minor role in explaining the degree of insincerity observed under PS in situation AH1. For all other situations, the frequency of insincere reporting does not differ significantly from that observed under PS. As in PS, in situations where there were many insincere reports, a large majority of them involved switching the top two choices.

Result 6. In situation AH1, non-sincere reporting is significantly less common under $R S D$ than under $P S$. 


\subsection{Efficiency under the PS vs. the RSD mechanism}

Although it was not the focus of our study, it is interesting to ask whether PS and RSD differ in terms of efficiency. In the three-agent case, ex post efficiency and ordinal efficiency are equivalent (Lemma 2 in Bogomolnaia and Moulin [5]). Thus both the mechanisms of PS and RSD are ordinally efficient, and there is no theoretical difference between the two. ${ }^{23}$ In order to compare efficiency of the two mechanisms in the experiment, we follow Chen and Sönmez [9] and compare the aggregate expected payoffs from the random assignment induced by the choices actually made between PS and RSD. ${ }^{24}$

For the symmetric games, the question of efficiency does not arise as any random assignment is ex ante efficient. Therefore we look only at the asymmetric games. Table 3 describes, for each situation in the game, the expected payoff from the Nash equilibrium outcome, and the average expected payoffs from choices in the experiments. The most prominent result visible in the table is:

Result 7. In game Asym-H, PS yields significantly larger aggregate expected utilities than RSD. In game Asym-L, the aggregate expected utilities of $P S$ and $R S D$ do not differ significantly.

This is driven by the pattern of misreporting documented in Section 4. As noted earlier, if players in situation AH1 misreport by switching their top choices while the others are sincere, the expected aggregate payoff is 295, as compared to 259 given the Nash equilibrium profile. This is true for both PS and RSD. Since this sort of switching behavior occurs significantly more often under PS, the realized aggregate payoff under that mechanism is substantially larger than the Nash equilibrium prediction, while the RSD payoff is rather close to the prediction. Looking at the individual player's payoffs we see that players AH1 achieve slightly lower expected payoffs under PS, while those in situation AH2 earn substantially more in expectation. Both differences, as well as the resulting difference in aggregate payoffs, are significant according to a two-sided Wilcoxon rank-sum test, using matching groups as units of observation. We see this result not as a recommendation of PS, since the higher payoffs are specific to the Asym-H game, but as a warning that Nash equilibrium may fail to predict the performance of matching mechanisms.

Turning to situation Asym-L, we find no significant difference in the aggregate payoffs under the two mechanisms, though there are significant differences in the payoffs achieved by individuals in situations AL1 and AL2. As in Asym-H, player 1's payoff is slightly smaller while player 2's is larger under PS. Though the difference is significant, it is not as pronounced, reflecting the fact that we see less misreporting here than in game Asym-H.

\footnotetext{
${ }^{23}$ When there are three agents, there is no room for agents to benefit from mutual exchanges of assignment probabilities. Note that when we demonstrated the ordinal inefficiency of RSD in Example 3, we had four agents.

${ }^{24} \mathrm{An}$ alternative approach would have been to look at the payoffs achieved as a result of the final assignments, after the resolution of uncertainty. However if this alternative method were to yield different results, such a difference would be the result of chance rather than features of the mechanisms themselves. Thus it is clear that expected payoffs are more informative than realized payoffs.
} 
Table 3: Average payoffs by situation and mechanisms

\begin{tabular}{ccccccc}
\hline Game and Situations & \multicolumn{2}{c}{ PS } & \multicolumn{2}{c}{ RSD } & \multicolumn{2}{c}{ PS vs. RSD } \\
& Nash & realized & Nash & realized & z-Stat & p value \\
\hline \multicolumn{7}{c}{ Game Asym-H } \\
AH1 (100,0,95) & 96.67 & 94.42 & 96.67 & 95.57 & -2.01 & 0.04 \\
AH2 (100,95,0) & 80.83 & 89.38 & 80.83 & 77.21 & 3.32 & $<0.01$ \\
AH3 (95,100,0) & 81.67 & 88.45 & 81.67 & 83.28 & 1.71 & 0.09 \\
Total in Asym-H & 259.17 & 272.26 & 259.17 & 256.06 & 3.32 & $<0.01$ \\
\hline \multicolumn{7}{c}{ Game Asym-L } \\
\hline AL1 (100,0,30) & 65 & 59.12 & 65 & 63.89 & -2.72 & $<0.01$ \\
AL2 (100,30,0) & 57.5 & 60.44 & 55 & 54.10 & 3.12 & $<0.01$ \\
AL3 (30,100,0) & 75 & 74.35 & 83.33 & 77.75 & -1.31 & 0.19 \\
Total in Asym-L & 197.5 & 193.91 & 203.33 & 195.74 & -0.91 & 0.36 \\
\hline
\end{tabular}

Note: Nash is the expected payoff in each situation under the Nash equilibrium. z statistics and p-values reflect a two-sided Wilcoxon rank-sum test using matching group averages.

\section{Conclusion}

We report the first laboratory experiment on the probabilistic serial mechanism (PS). While PS is not strategy-proof, we were not able to observe strategic misrepresentation of preferences in situations where it would have benefited agents. However, we did find high levels of misrepresentation in a situation where this was not appropriate. This behavior did not become less prevalent with subjects' experience. We believe that the behavior may be caused by subjects wrongly applying a heuristic of avoiding popular options.

We conclude that the design of matching mechanisms, like auction design, needs to be sensitive to behavioral anomalies as well as to optimal strategic behavior. This could be a concern for the use of the mechanism in real-world situations, particularly because, while PS becomes strategy-proof in large enough economies, this does not protect against mistaken strategic behavior.

\section{Acknowledgements}

We would like to thank Onur Kesten, M. Utku Ünver, and two referees for their comments. Kurino acknowledges the research support of Maastricht University when he was affiliated there. We acknowledge the financial support from Maastricht University, the Netherlands Organisation for Scientific Research (NWO) under grand VIDI-452-06-013, and the University of Heidelberg.

[1] AbdulkadiroĞlu, A., P. A. Pathak, A. E. Roth, and T. Sönmez (2005): "The Boston Public School Match," American Economic Review Papers and Proceedings, 95, 368-372.

[2] AbdulkadiroĞlu, A., And T. Sönmez (1999): "House Allocation with Existing Tenants," Journal of Economic Theory, 88, 233-260. 
[3] (2003): "School Choice: A Mechanism Design Approach," American Economic Review, 93, 729-747.

[4] Birkhoff, G. (1946): "Three Observations on Linear Algebra," Revi. Univ. Nac. Tucuman, ser $A, 5,147-151$.

[5] Bogomolnaia, A., And H. Moulin (2001): "A New Solution to the Random Assignment Problem," Journal of Economic Theory, 100, 295-328.

[6] Budish, E., and E. Cantillon (2012): "The Multi-unit Assignment Problem: Theory and Evidence from Course Allocation at Harvard," American Economic Review, 102(5), 2237-2271.

[7] Chen, Y., And T. Sönmez (2002): "Improving Efficiency of On-campus Housing: An Experimental Study," American Economic Review, 92, 1669-1686.

[8] (2004): "An Experimental Study of House Allocation Mechanisms," Economics Letters, $83,137-140$.

[9] - (2006): "School Choice: An Experimental Study," Journal of Economic Theory, 127, 202-231.

[10] Ekici, ÖzGün., And O. Kesten (2012): "An Equilibrium Analysis of the Probabilistic Serial Mechanism," Working paper.

[11] Fischbacher, U. (2007): "z-Tree: Zurich Toolbox for Ready-made Economic Experiments," Experimental Economics, 10(2), 171-178.

[12] Foley, D. (1967): "Resource Allocation and the Public Sector," Yale Economic Essays, 7, 45-98.

[13] Guillen, P., and A. Hing (2013): "Lying through Their Teeth: Third Party Advice and Truth Telling in a Strategy Proof Mechanism," Working paper.

[14] Guillen, P., and O. Kesten (2012): "Matching Markets with Mixed Ownership: The Case for a Real-life Mechanism," International Economic Review, 53(3), 1027-1046.

[15] Hashimoto, T., D. Hirata, O. Kesten, M. Kurino, and M. U. Ünver (2012): "Two Axiomatic Approaches to the Probabilistic Serial Mechanism," Forthcoming, Theoretical Economics.

[16] Hylland, A., and R. Zeckhauser (1979): "The Efficient Allocation of Individuals to Positions," Journal of Political Economy, 87, 293-314.

[17] Kesten, O. (2009): "Why Do Popular Mechanisms Lack Efficiency in Random Environments?," Journal of Economic Theory, 144, 2209-2226. 


\begin{tabular}{ccc} 
& $(1)$ & $(2)$ \\
\cline { 2 - 3 } Constant & 0.1628 & 0.2087 \\
& $(0.2885)$ & $(0.2463)$ \\
Repetition & $0.1345+$ & \\
& $(0.0760)$ & $0.0163+$ \\
Period & & $(0.0090)$ \\
& & 396 \\
$\mathrm{~N}$ & 396 & 66 \\
$\mathrm{~N}$ indiv. & 66 & 5.00 \\
Chi-sq & 4.69 & $\mathrm{par}$
\end{tabular}

Standard errors clustered by individual in parentheses. $+\mathrm{p}<0.10$

Table 4: Logistic regressions of insincerity on repetition and on period

[18] Kesten, O., And A. Yazici (2012): "The Pareto Dominant Strategy-Proof and Fair Rule for Problems with Indivisible Goods," Economic Theory, 50 (2).

[19] Kojima, F., And M. Manea (2010): "Incentives in the Probabilistic Serial Mechanism," Journal of Economic Theory, 144, 106-123.

[20] Roth, A. E., T. Sönmez, And M. U. Ünver (2004): "Kidney Exchange," Quarterly Journal of Economics, 119, 457-488.

[21] Shapley, L., And H. SCARF (1974): "On Cores and Indivisibility," Journal of Mathematical Economics, 1, 23-37.

[22] SÖnmez, T., And M. U. Ünver (2010): "Course Bidding at Business Schools," International Economic Review, 51, 99-123.

[23] Von Neumann, J. (1953): "A Certain Zero-sum Two-person Game Equivalent to the Optimal Assignment Problem," in Contributions to the theory of games, Vol. 2, ed. by H. W. Kuhn, and A. W. Tucker. Princeton University Press, Princeton, New Jersey.

\section{Appendix}

Table 4 shows logistic regressions confirming that insincerity does not disappear over time. The dependent variable is a dummy taking the value 1 if the subject switched her top two options. The regressions show no significant change in insincerity when the independent variable is the repetition of the situation (i.e. the number of times the subject has seen the specific situation, from 1 to 6 ), and a marginally significant increase in insincerity when the independent variable is the period (from 1 to 48). If we instead use a dummy for any kind of insincerity (not just switching) as a dependent variable, this significance vanishes. 